\title{
Robust MPC of an unstable chemical reactor using the nominal system optimization
}

\author{
Monika Bakošová, Juraj Oravec \\ Slovak University of Technology in Bratislava, Faculty of Chemical and Food Technology, \\ Institute of Information Engineering, Automation, and Mathematics, \\ Radlinského 9, SK-812 37 Bratislava, Slovak Republic \\ monika.bakosova@stuba.sk
}

\begin{abstract}
The continuous stirred-tank reactor with uncertain parameters was stabilized in the open-loop unstable steady state using the robust model predictive control. The gain matrices of the robust state-feedback controller were designed using the nominal system optimization and the quadratic parameter-dependent Lyapunov functions. The controller was verified by simulations using the non-linear model of the reactor and compared with the robust model predictive controller designed using the worst-case system optimization. The values of the quadratic cost function and the consumption of coolant were observed. Both robust model predictive controllers stabilized the reactor despite constrained control inputs and states. The robust model predictive control based on the nominal system optimization improved control responses and decreased the consumption of coolant.
\end{abstract}

Keywords: chemical reactor, uncertainty, robust MPC, linear matrix inequality, Lyapunov function

\section{Introduction}

Chemical reactors are very important equipment in chemical and food industries, and they are complex and complicated systems from the control viewpoint. The main reasons are the nonlinear behaviour (Kvas-

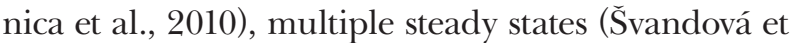
al., 2005), potential safety threats in reactors with exothermic chemical reactions (Ball and Gray, 2013) or presence of various uncertainties. Some of them arise from varying or not exactly known parameters, as e.g. reaction enthalpies, reaction rate constants, heat transfer coefficients, etc. (Laššák, 2010; Bakošová, 2012). These uncertainties can cause low quality of the control performance or even instability of the closed-loop controlled system. Implementation of a robust model-based predictive control (MPC) strategy designed using the Lyapunov functions overcomes these problems. The advantage of this approach is handling uncertainty, input and output constraints and getting optimal solution in each control step. The disadvantage is the significant computational burden necessary for solving the convex optimization problem that is often formulated using linear matrix inequalities (Kothare et al., 1996). Wu (2001) designed the robust MPC based on the state feedback and the worst-case objective function optimisation for a class of systems with structured time-varying uncertainty and presented implementation of the designed robust MPC to the stable CSTR control problem. To reduce conservatism Ding (2010) designed a parameterdependent dynamic output feedback and proposed an iterative algorithm for the on-line synthesis of the control law via convex optimization. Ghaffari et al.
(2013) extended the robust MPC design for additive discrete time uncertain nonlinear systems, designed the controller using the worst-case optimisation and presented the simulation results obtained for the theoretical CSTR with the first order chemical reaction working in the stable operating point.

Based on previous works (Bakošová et al., 2013, Bakošová and Oravec, 2013; Oravec and Bakošová, 2012), this paper studies the problem of stabilization of an open-loop unstable CSTR using the robust constrained MPC based on the nominal system optimization and the parameter-dependent Lyapunov functions (PDLFs). This approach was compared with the robust MPC designed using the worst-case system optimization. The conditions for the robust MPC design were formulated in the form of linear matrix inequalities (LMIs) in both approaches. Solution of the LMIs represents a convex optimization problem that was solved in the MATLAB environment by the YALMIP toolbox (Löfberg, 2004) with the SeDuMi solver (Sturm, 1999) and the non-iterative algorithm was used. The robust stabilization of the CSTR was simulated using the non-linear model.

\section{Theoretical}

Suppose that the controlled process is a linear statespace system in Eq. (1)

$$
\begin{aligned}
& x(k+1)=A(k) x(k)+B(k) u(k), \quad x(0)=x_{0} \\
& y(k)=C x(k) \\
& {[A(k), B(k)] \in \Omega} \\
& \Omega=\operatorname{conv}\left\{\left[A^{(v)}, B^{(v)}\right]\right\}, \quad v \in\left\{1, \ldots, N_{v}\right\}, \quad k \geq 0
\end{aligned}
$$


where $k$ is the discrete time, $x(k)$ is the vector of system states, $u(k)$ is the control input vector, $y(k)$ is the controlled output vector and the matrices $A(k)$, $B(k), C$ have appropriate dimensions. $A^{(v)}, B^{(v)}$ are matrices of the $v$-th vertex of the uncertain system. $\Omega$ is a convex set of all admissible controlled systems and conv is a function returning the convex hull of the system vertices.

The symmetric control input constraints are in the form of the Euclidean and the peak norms and the symmetric controlled output constraints are in the form of the Euclidean norm in Eq. (2)

$$
\begin{gathered}
\|u(k)\|_{2} \leq u_{\max }, \quad\left|u_{j}(k)\right| \leq u_{j, \max }, \quad j \in\left\{1,2, \ldots, N_{u}\right\}, \\
\|y(k)\|_{2} \leq y_{\max }, \quad k \geq 0
\end{gathered}
$$

The task is to design a state feedback controller that assures the stability of the closed loop with the unstable controlled system in Eq. (1). The statefeedback control law is described by Eq. (3)

$$
u(k)=F(k) x(k)
$$

where $F(k)$ is the gain matrix of the robust statefeedback controller in the $k$-th control step. The simplified notation of the discrete-time dependence in the form $F_{k}=F(k)$ will be used in the next text.

To design the gain matrix $F_{k}$, the approach described in Cuzzola et al. (2002) was applied. The conditions in Eq. (4) hold for the square parameter depended Lyapunov matrix $P_{k}^{(v)}=\left(P_{k}^{(v)}\right)^{\mathrm{T}}>0$, the inverse Lyapunov matrix $Q_{k}$, the inverse parameterdependent Lyapunov matrix $X_{k}^{(v)}=\left(X_{k}^{(v)}\right)^{\mathrm{T}}>0$, the auxiliary matrix $Y_{k}$, and the weight parameter $\gamma_{k}$.

$$
\begin{gathered}
X_{k}^{(v)}=\gamma_{k}\left(P_{k}^{(v)}\right)^{-1}, \quad v \in\left\{1, \ldots, N_{v}\right\}, \\
Y_{k}=F_{k} Q_{k} \Rightarrow F_{k}=Y_{k} Q_{k}^{-1}
\end{gathered}
$$

The quadratic cost function in Eq. (5) evaluates the quality of control from the $k$-th to the $N$-th control step

$$
J=t_{\mathrm{s}} \sum_{i=k}^{N}\left(x_{k+i}^{\mathrm{T}} W_{x} x_{k+i}+u_{k+i}^{\mathrm{T}} W_{u} u_{k+i}\right)
$$

where $t_{\mathrm{s}}$ is the sampling time and $W_{x}, W_{u}$ are the real symmetric weight matrices. The minimization of $J$ assures the optimal solution of the robust MPC problem that can be transformed into the solution of a convex optimization problem formulated using the linear matrix inequalities (LMIs) in Eqs. (6)-(8).

$$
\min _{Q_{k}, X_{k}^{(v)}, Y_{k}} \gamma_{k}
$$

subject to

$$
\left[\begin{array}{cc}
1 & x_{k}^{\mathrm{T}} \\
* & X_{k}^{(v) \mathrm{T}}
\end{array}\right] \geq 0, \quad v \in\left\{1, \ldots, N_{v}\right\}
$$

$$
\left[\begin{array}{cccc}
Q_{k}+Q_{k}^{\mathrm{T}}-X_{k}^{(v) T} & Q_{k}^{\mathrm{T}} A^{(w) \mathrm{T}}+Y_{k}^{\mathrm{T}} B^{(w) \mathrm{T}} & Q_{k}^{\mathrm{T}} \sqrt{W_{x}} & Y_{k}^{\mathrm{T}} \sqrt{W_{u}} \\
* & X_{k}^{(v)} & 0 & 0 \\
* & * & \gamma_{k} I & 0 \\
* & * & * & \gamma_{k} I
\end{array}\right] \geq 0,
$$

The LMIs are obtained using substitutions and Schur complement formula (Cuzzola et al., 2002). Minimization of $J$ for $N \rightarrow \infty$ in Eq. (5) is transformed to minimization of the auxiliary weight parameter $\gamma$ in Eq. (6), that assures minimization of the weight of the inverse parameter-dependent Lyapunov matrix $X_{k}^{(v)}$ of the robust stability condition. The symbol * in Eq. (8) denotes the symmetric structure of the matrix, $I$ and 0 represent identity and zero matrices of appropriate dimensions, respectively. Parameters $v$ and $w$ are the indices of the system vertices, $\Phi$ is the set of vertex indices. Several approaches were designed for solution of the convex optimisation problems formulated using LMIs. Cuzzola et al. (2002) designed the standard worst-case system optimization approach (WCSOA). The improvement of the algorithm is based on the nominal system optimization approach (NSOA) (Ding et al., 2007) of the LMI in Eq. (8). Two different approaches for robust MPC design are used also in this paper. The first one is the standard WCSOA for the set $w \in$ $\Phi=\left\{1, \ldots, N_{v}\right\}$, which designs the Lyapunov matrix using all system vertices. The second approach is the NSOA that uses the singleton $w \in \Phi=\{0\}$ and constructs the Lyapunov matrix taking into account only the nominal system. The constraints on control inputs in Eq. (2) can be included to the convex optimization problem in Eqs. (6)-(8) by adding the LMIs in Eq. (9)

$$
\begin{gathered}
{\left[\begin{array}{cc}
u_{\max }^{2} I & Y_{k} \\
* & Q_{k}+Q_{k}^{\mathrm{T}}-X_{k}^{(v)}
\end{array}\right]} \\
{\left[\begin{array}{cc}
U_{k} & Y_{k} \\
* & Q_{k}+Q_{k}^{\mathrm{T}}-X_{k}^{(v)}
\end{array}\right]} \\
U_{j, j}(k) \leq u_{j, \max }^{2}, \quad j=\left\{1, \ldots, N_{u}\right\}, \quad v \in\left\{1, \ldots, N_{v}\right\}
\end{gathered}
$$

Similarly, the constraints on the controlled outputs can be incorporated into the robust MPC design adding the LMIs in Eq. (10)

$$
\begin{aligned}
& {\left[\begin{array}{cc}
Q_{k}+Q_{k}^{\mathrm{T}}-X_{k}^{(v)} & \left(A^{(v)} Q_{k}+B^{(v)} Y_{k}\right)^{\mathrm{T}} C^{\mathrm{T}} \\
* & y_{\max }^{2} I
\end{array}\right] \geq 0,} \\
& v \in\left\{1, \ldots, N_{v}\right\}
\end{aligned}
$$

The convex optimization problem is solved in each control step $k$. Therefore it is necessary to use proper sampling time $t_{\mathrm{s}}$. 
The algorithm of the robust constrained MPC has following steps:

1. Decide whether the WCSOA set of system vertices $\Phi=\left\{1, \ldots, N_{v}\right\}$ or the NSOA singleton $\Phi=\{0\}$ is used.

2. Set $k=0$ and set the initial values of the sampling time $t_{\mathrm{s}}$, the weight matrices $W_{x}, W_{u}$, the total number of control steps $N$, the initial conditions of the system states $x(0)$, the values of the boundaries on the control inputs $u_{\max }$ and the controlled outputs $y_{\max }$.

3. Update the control step $k=k+1$.

4. Measure or estimate the current values of the system states $x(k)$.

5. Solve the convex optimization problem described by Eqs. (6) - (10) and find the matrices $Q_{k}, X_{k}^{(v)}$ and $Y_{k}$.

6. Find the gain matrix $F_{k}$ of the state-feedback robust model predictive controller using Eq. (4).

7. Calculate the control input $u(k)$ using Eq. (3).

8. Use calculated control input $u(k)$ for control of the system in Eq. (1).

9. If $k<N$ then go to third step else stop.

\section{Experimental}

The controlled process is a continuous-time stirredtank reactor (CSTR) for hydrolysis of propylene oxide $\left(\mathrm{C}_{3} \mathrm{H}_{6} \mathrm{O}\right)$ to propylene glycol $\left(\mathrm{C}_{3} \mathrm{H}_{8} \mathrm{O}_{2}\right)$ (Molnár et al., 2002) according to the Eq. (11)

$$
\mathrm{C}_{3} \mathrm{H}_{6} \mathrm{O}+\mathrm{H}_{2} \mathrm{O} \stackrel{\mathrm{CH}_{3} \mathrm{OH}}{\longrightarrow} \mathrm{C}_{3} \mathrm{H}_{8} \mathrm{O}_{2}, \quad \Delta_{\mathrm{r}} H<0
$$

The first order exothermic chemical reaction is performed in a reaction vessel and the reaction heat is withdrawn from the reactor by the coolant in

Tab. 1. Technological parameters of the CSTR.

\begin{tabular}{lc}
\hline Parameter $/$ Unit & Value \\
\hline$V_{\mathrm{r}} / \mathrm{m}^{3}$ & 2.4 \\
$V_{\mathrm{c}} / \mathrm{m}^{3}$ & 2.0 \\
$\rho_{\mathrm{r}} /\left(\mathrm{kg} \mathrm{m}^{-3)}\right.$ & 947.19 \\
$\rho_{\mathrm{c}} /\left(\mathrm{kg} \mathrm{m}^{-3}\right)$ & 998.00 \\
$c_{P, \mathrm{r}} /\left(\mathrm{kJ} \mathrm{kg}^{-1} \mathrm{~K}^{-1}\right)$ & 3.719 \\
$C_{P, \mathrm{c}} /\left(\mathrm{kJ} \mathrm{kg}^{-1} \mathrm{~K}^{-1}\right)$ & 4.182 \\
$A_{\mathrm{h}} / \mathrm{m}^{2}$ & 8.695 \\
$g=\left(E_{\mathrm{a}} / R_{g}\right) / \mathrm{K}$ & 10183.0 \\
\hline
\end{tabular}

the reactor jacket. Technological parameters of the CSTR are given in the Table 1 .

Model uncertainties of the reactor follow from the fact that there are three only approximately known physical parameters, which minimal, nominal and maximal values are shown in Table 2. Here, $\Delta_{\mathrm{r}} H$ is the reaction enthalpy of the chemical reaction, $k_{\mathrm{r}, \infty}$ is the pre-exponential factor in the reaction rate constant and $U_{\mathrm{h}}$ is the heat transfer coefficient. The nominal values of the uncertain parameters are the mean values of given intervals. Therefore the structured interval parametric uncertainties are considered. The multivariable controller design is assumed in order to assure control of the reaction mixture temperature in the reaction vessel $T_{\mathrm{r}}(t)$ and the coolant temperature $T_{\mathrm{c}}(t)$ in the jacket. Control inputs are the volumetric flow rates of the reaction mixture $q_{\mathrm{r}}(t)$ and the coolant $q_{\mathrm{c}}(t)$.

The nonlinear model of the CSTR is obtained by the mass balances of the propylene oxide (PO) and the main product propylene glycol (PG), and by the enthalpy balances of the reaction mixture and the coolant. The exponential dependence of the reaction rate $k_{\mathrm{r}}(t)$ on the temperature of the reaction mixture $T_{\mathrm{r}}(t)$ is described by the Arrhenius equation in the form $k_{\mathrm{r}}(t)=k_{\mathrm{r}, \infty} \exp \left(-g / T_{\mathrm{r}}(t)\right)$. Applying simplifying assumptions the mathematical model of the CSTR can be described using four non-linear differential equations in Eqs. (12)-(15)

$$
\begin{aligned}
& \frac{\mathrm{d} c_{\mathrm{PO}}(t)}{\mathrm{d} t}=\frac{q_{\mathrm{r}}(t)}{V_{\mathrm{r}}} c_{\mathrm{PO}, \text { in }}-\left(\frac{q_{\mathrm{r}}(t)}{V_{\mathrm{r}}}+k_{\mathrm{r}}(t)\right) c_{\mathrm{PO}}(t) \\
& \frac{\mathrm{d} c_{\mathrm{PG}}(t)}{\mathrm{d} t}=\frac{q_{\mathrm{r}}(t)}{V_{\mathrm{r}}} c_{\mathrm{PG}, \text { in }}-\frac{q_{\mathrm{r}}(t)}{V_{\mathrm{r}}} c_{\mathrm{PG}}(t)+k_{\mathrm{r}}(t) c_{\mathrm{PO}}(t)
\end{aligned}
$$

$$
\begin{gathered}
\frac{\mathrm{d} T_{\mathrm{r}}(t)}{\mathrm{d} t}=-\frac{k_{\mathrm{r}}(t) \Delta_{\mathrm{r}} H}{\rho_{\mathrm{r}} c_{P, \mathrm{r}}} c_{P O}(t)+ \\
+\frac{q_{\mathrm{r}}(t)}{V_{\mathrm{r}}}\left(T_{\mathrm{r}, \mathrm{in}}-T_{\mathrm{r}}(t)\right)+\frac{U_{\mathrm{h}} A_{\mathrm{h}}}{V_{\mathrm{r}} \rho_{\mathrm{r}} c_{P, \mathrm{r}}}\left(T_{\mathrm{r}}(t)-T_{\mathrm{c}}(t)\right) \\
\frac{\mathrm{d} T_{\mathrm{c}}(t)}{\mathrm{d} t}=\frac{q_{\mathrm{c}}(t)}{V_{\mathrm{c}}}\left(T_{\mathrm{c}, \mathrm{in}}-T_{\mathrm{c}}(t)\right)+\frac{U_{\mathrm{h}} A_{\mathrm{h}}}{V_{\mathrm{c}} \rho_{\mathrm{c}} c_{P, \mathrm{c}}}\left(T_{\mathrm{r}}(t)-T_{\mathrm{c}}(t)\right)
\end{gathered}
$$

Tab. 2. Uncertain parameters of the CSTR.

\begin{tabular}{lccc}
\hline Parameter $/$ Unit & Minimal Value & Nominal Value & Maximal Value \\
\hline$\Delta_{\mathrm{r}} H /\left(\mathrm{kJ} \mathrm{kmol}^{-3}\right)$ & $-5.64 \times 10^{6}$ & $-5.34 \times 10^{6}$ & $-5.28 \times 10^{6}$ \\
$k_{\mathrm{r}, \infty} /\left(\mathrm{min}^{-1}\right)$ & $2.4067 \times 10^{11}$ & $2.8267 \times 10^{11}$ & $3.2467 \times 10^{11}$ \\
$U_{\mathrm{h}} /\left(\mathrm{kJ} \mathrm{min}^{-1} \mathrm{~m}^{-2} \mathrm{~K}^{-1}\right)$ & 13.11 & 13.80 & 14.49 \\
\hline
\end{tabular}


Hence, the CSTR is the fourth-order nonlinear system with two control inputs $q_{r}, q_{\mathrm{c}}$ and four states $c_{\mathrm{PO}}, c_{\mathrm{PG}}, T_{\mathrm{r}}, T_{\mathrm{c}}$. The steady-state analysis of the reactor is described in Oravec and Bakošová, 2012. The CSTR has three steady states. The temperatures of the reaction mixture $296.7 \mathrm{~K}$ and $377.5 \mathrm{~K}$ correspond to the stable steady-states and the temperature $T_{r}^{\mathrm{s}}=343.1 \mathrm{~K}$ refers to the unstable steady state. From the control view-point the exothermic reaction can represent a potential safety problem. Therefore the possibility to use the robust MPC for stabilisation of the CSTR into the unstable steady state was investigated. The considered inlet values, the unstable steady-state values and the initial values are given in Table 3 .

The nonlinear model of the CSTR described by Eqs. (12)-(15) was linearized in the unstable operating point and transformed from the continuoustime domain into the discrete-time domain using the sampling time $t_{\mathrm{s}}=0.5 \mathrm{~min}$. The state-space model in the form of Eq. (1) was obtained, where vectors $x(k), u(k)$ and $y(k)$ are defined in Eq. (16)

$$
\begin{aligned}
& x(k)=\left[\begin{array}{c}
c_{\mathrm{PO}}(k)-c_{\mathrm{PO}}^{\mathrm{s}} \\
c_{\mathrm{PG}}(k)-c_{\mathrm{PG}}^{\mathrm{s}} \\
T_{\mathrm{r}}(k)-T_{\mathrm{r}}^{\mathrm{s}} \\
T_{\mathrm{c}}(k)-T_{\mathrm{c}}^{\mathrm{s}}
\end{array}\right], \quad u(k)=\left[\begin{array}{c}
q_{\mathrm{r}}(k)-q_{\mathrm{r}}^{\mathrm{s}} \\
q_{\mathrm{c}}(k)-q_{\mathrm{c}}^{\mathrm{s}}
\end{array}\right], \\
& y(k)=x(k)
\end{aligned}
$$

The superscript $s$ denotes the steady-state value. As three of the technological parameters are uncertain (Table 2), it is possible to obtain $N_{v}=2^{3}=8$ different vertex systems using all combinations of the boundary values of uncertain parameters. The convex hull of the system vertices describes the admissible range of the CSTR behaviour. The $9^{\text {th }}$ system is the nominal model of the CSTR evaluated for the mean values of uncertain parameters $\Delta_{\mathrm{r}} H, k_{\mathrm{r}, \infty}$, and $U_{\mathrm{h}}$. The nominal system is considered as the reference system.

\section{Results and discussion}

The closed-loop stabilization of the CSTR into its unstable steady state using the robust MPC was studied. Both, WCSOA and NSOA were used for the robust MPC state-feedback controller design in the MATLAB-Simulink environment. The optimization problem in Eqs. (6)-(10) was solved using the YALMIP toolbox (Löfberg, 2004) with the SeDuMi solver (Sturm, 1999) and the 3.20 GHz CPU and the memory 4 GB RAM were used. The weight matrices in the cost function in Eq. (5) were the same in both approaches to make the results comparable and they were in the form of diagonal matrices with the main diagonals $\operatorname{diag}\left(W_{x}\right)=[0.01,0.01,100,100]^{\mathrm{T}}, \operatorname{diag}\left(W_{u}\right)=[1,1]$ ${ }^{\mathrm{T}}$ and zeros elsewhere. The symmetric boundaries in Eq. (2) were calculated for $q_{\mathrm{r}, \max }=0.14 \mathrm{~m}^{3} \mathrm{~min}^{-1}$, $q_{\mathrm{c}, \max }=1.26 \mathrm{~m}^{3} \mathrm{~min}^{-1}, T_{\mathrm{r}, \max }=373.1 \mathrm{~K}, T_{\mathrm{c}, \max }=320.6 \mathrm{~K}$ using Eq. (16). Both robust MPC strategies were compared by simulation using the nonlinear model of the CSTR. The behaviour of eight vertex systems and the nominal system was investigated. To show clearly the difference between the approaches, only the worst and the best control trajectories of all nine possible ones are depicted in the Figures 1, 2. The Figure 1a) shows the controlled outlet temperature of the reaction mixture $T_{\mathrm{r}}(t)$ assured by the WCSOA (dashed) and NSOA (solid) strategy, respectively. The worst control response (squares) is compared with the best control response (circles). The refer-

Tab. 3. Inlet values, steady-state values and initial values of variables in the nominal CSTR.

\begin{tabular}{lccc}
\hline Variable $/$ Unit & Inlet Value & Steady-state Value & Initial Value \\
\hline$c_{\mathrm{PO}} /\left(\mathrm{kmol} \mathrm{m}^{-3}\right)$ & 0.082 & 0.037 & 0.042 \\
$c_{\mathrm{PG}} /\left(\mathrm{kmol} \mathrm{m}^{-3}\right)$ & 0.000 & 0.045 & 0.040 \\
$T_{\mathrm{r}} / \mathrm{K}$ & 299.1 & 343.1 & 341.1 \\
$T_{\mathrm{c}} / \mathrm{K}$ & 288.2 & 290.6 & 288.6 \\
$q_{\mathrm{r}} /\left(\mathrm{m}^{3} \mathrm{~min}^{-1}\right)$ & - & 0.072 & 0.072 \\
$q_{\mathrm{c}} /\left(\mathrm{m}^{3} \mathrm{~min}^{-1}\right)$ & - & 0.631 & 0.631 \\
\hline
\end{tabular}

Tab. 4. The cost function values and the coolant consumption.

\begin{tabular}{lllllll}
\hline robust MPC approach: & WCSOA & \multicolumn{3}{c}{ Comparison } \\
\hline System & $V_{\text {c,total }}{ }^{\text {WSOA }} / \mathrm{m}^{3}$ & $J^{\text {WCSOA }}$ & $V_{\text {c,total }}{ }^{\mathrm{NSOA}} / \mathrm{m}^{3}$ & $J^{\mathrm{NSOA}}$ & $\Delta V_{c, \text { total }} / \%$ & $\Delta J / \%$ \\
\hline Mean case & 5.155 & 1071.6 & 4.893 & 959.7 & 5.1 & 10.4 \\
Worst case & 5.482 & 1301.9 & 5.217 & 1121.7 & 4.8 & 13.8 \\
Best case & 4.831 & 870.4 & 4.583 & 814.2 & 5.1 & 6.5 \\
\hline
\end{tabular}


ence value is the temperature of the reaction mixture $T_{\mathrm{r}}^{\mathrm{s}}=343.1 \mathrm{~K}$ (dotted line). The Figure 1b) shows the controlled outlet temperature of the coolant $T_{c}(t)$. Here, the unstable operating point refers to $T_{\mathrm{c}}^{\mathrm{s}}=290.6 \mathrm{~K}$ (dotted). In both cases the temperatures converge to the unstable operating point. Using the NSOA improved control responses. As can be seen in Figure 1, the settling time decreased. The offsets originate from the fact, that the linear model was used for the controller design and the non-linear model of the CSTR was controlled. The offset-free behaviour was obtained when the linear model of the CSTR was controlled (Bakošová and Oravec, 2013). The Figure 2 compares the control inputs - the volumetric flow-rate of the reaction mixture $q_{\mathrm{r}}(t)$ and the volumetric flow-rate of the cooling medium $q_{\mathrm{c}}(t)$. The values of the volumetric flow rates $q_{\mathrm{r}}(t)$ and $q_{\mathrm{c}}(t)$ calculated from Eq. (16)

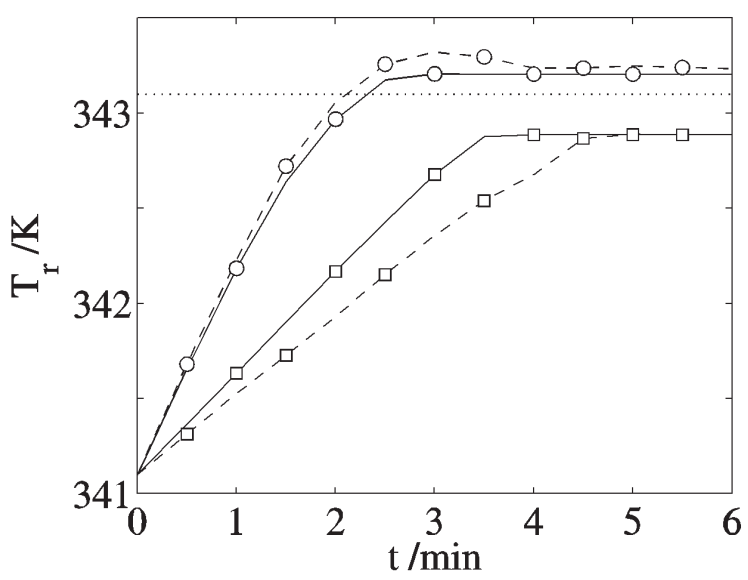

a) stayed within the prescribed constraints. The value of the cost function $J$ and the total consumption of the coolant $V_{\text {ctotal }}$ were also evaluated and the results are presented in Table 4 . The maximal and the minimal values denote the worst and the best situation, respectively. The values $\Delta V_{\mathrm{c} \text {,total }}$ and $\Delta J$ were computed using Eq. (17) that represent the coolant reduction and the quality improvement using the NSOA. The NSOA reduced consumption of the coolant in about $5 \%$ and improved the quality represented by the value of $J$ in Eq. (5) from 6.5 to $14 \%$.

$$
\begin{gathered}
\Delta V_{\mathrm{c}, \text { total }}=\left(1-\frac{V_{\mathrm{c}, \text { total }}^{N S O A}}{V_{\mathrm{c}, \text { total }}^{\mathrm{WCSOA}}}\right) \times 100 \%, \\
\Delta J=\left(1-\frac{J^{N S O A}}{J^{\mathrm{WCSOA}}}\right) \times 100 \%
\end{gathered}
$$

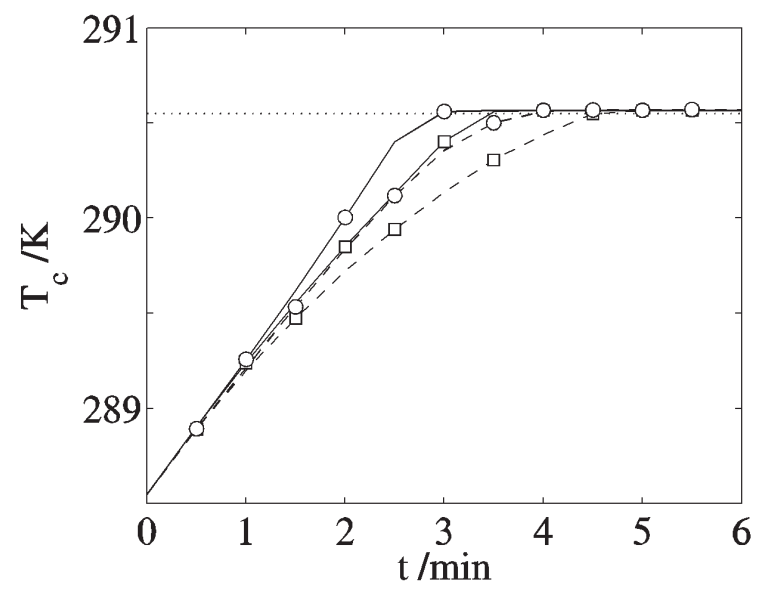

b)

Fig. 1. (a) The best (o) and the worst $(\square)$ control responses of the reaction mixture temperature $T_{\mathrm{r}}(t)$ and (b) the best (o) and the worst $(\square)$ control responses of the cooling medium $T_{c}(t)$ assured by the WCSOA (- - -) and the NSOA (-).

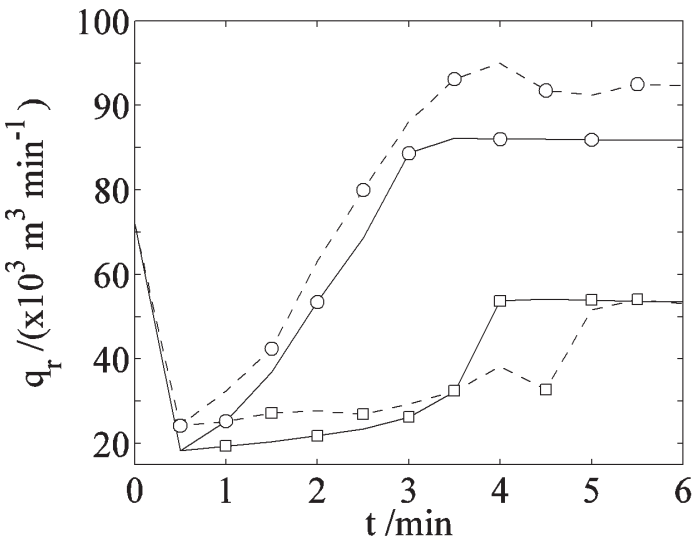

a)

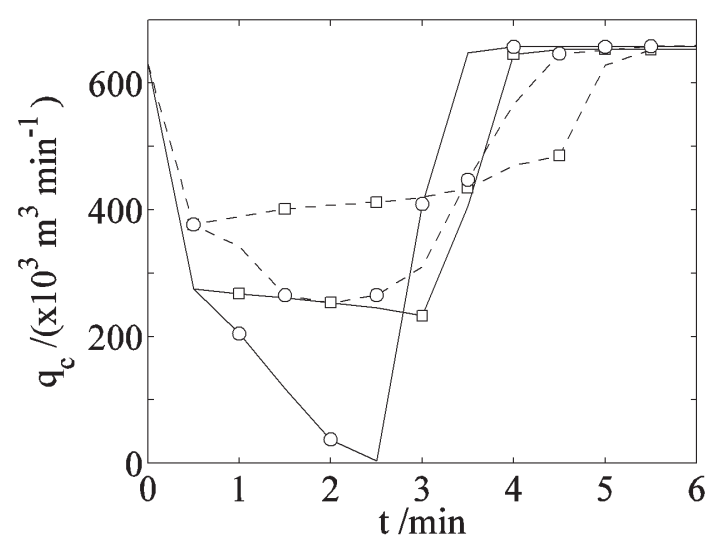

b)

Fig. 2. (a) The best (o) and the worst $(\square)$ control input trajectories of the volumetric flow rates of reaction mixture $q_{\mathrm{r}}(t)$ and (b) the best $(\mathrm{o})$ and the worst $(\square)$ control input trajectories of the cooling medium $q_{C}(t)$ assured by the WCSOA (- - -) and the NSOA (-). 


\section{Conclusions}

The robust constrained model predictive control of the CSTR was studied. The complexity of the controller design originated from the fact that the CSTR was described by eight vertex systems, and all of them had to be stabilized simultaneously. Moreover, the behaviour of the CSTR was nonlinear. The robust state-feedback controllers were designed using two approaches. The WCSOA considered eight vertex systems and the NSOA considered only the nominal system for optimisation. The controller design procedures were noniterative. The NSOA reduced the conservativeness and from the computational viewpoint simplified the solved problem. The obtained simulation results confirmed that the robust controller designed using the NSOA improved control performances and the coolant consumption was smaller in comparison with the WCSOA. Improvement of the NSOA design that will assure the offset-free control responses of the non-linear system will be the subject of the next research.

\section{Acknowledgement}

This article was created with the support of the Ministry of Education, Science, Research and Sport of the Slovak Republic within the Research and Development Operational Programme for the project "University Science Park of STU Bratislava”, ITMS 26240220084, co-funded by the European Regional Development Fund.

\section{Symbols}

A state-space model matrix of states

$A_{h} \quad$ heat exchange surface area

$B \quad$ state-space model matrix of inputs

C state-space model matrix of outputs

$c$

$c_{P}$

$\Delta_{\mathrm{r}} H$

$E_{a}$ molar concentration $\quad \mathrm{kmol} \mathrm{m}^{-3}$ reaction enthalpy $\quad \mathrm{kJ} \mathrm{kmol}^{-1} \mathrm{~min}^{-1}$ activation energy $\quad \mathrm{kJ} \mathrm{kmol}^{-1} \mathrm{~min}^{-1}$ gain matrix of state feedback controller quadratic cost function relative quadratic cost function value \% control step reaction rate pre-exponential factor $\mathrm{kmol} \min ^{-1}$ $\min ^{-1}$ number of control steps number of system inputs number of uncertain system vertices number of system states number of system outputs auxiliary matrix of quadratic stability criteria volumetric flow rate

$\mathrm{m}^{3} \min ^{-1}$

\begin{tabular}{|c|c|}
\hline$R_{g}$ & universal gas constant \\
\hline$t$ & $\mathrm{~min}$ \\
\hline$U$ & $\begin{array}{l}\text { auxiliary matrix of inputs of robust } \\
\text { model predictive control }\end{array}$ \\
\hline$U_{\mathrm{h}}$ & heat transfer coefficient $\mathrm{kJ} \min ^{-1} \mathrm{~m}^{-2} \mathrm{~K}^{-1}$ \\
\hline$u$ & vector of system inputs \\
\hline$V$ & volume \\
\hline$V_{\mathrm{c} \text { total }}$ & total consumption of coolant \\
\hline$\Delta V_{\text {c,total }}$ & relative consumption of coolant \\
\hline$v$ & index of uncertain system vertex \\
\hline$W_{u}$ & cost function weight matrix of inputs \\
\hline$W_{x}$ & cost function weight matrix of states \\
\hline$w$ & index of uncertain system vertex \\
\hline$X$ & $\begin{array}{l}\text { weighted inverse parameter-dependent } \\
\text { Lyapunov matrix }\end{array}$ \\
\hline$x$ & vector of system states \\
\hline$Y$ & $\begin{array}{l}\text { auxiliary matrix of robust model } \\
\text { predictive controller design }\end{array}$ \\
\hline & vector of system outputs \\
\hline
\end{tabular}

\section{Greek Letters}

$\gamma \quad$ optimized variable of robust model predictive control

$\rho$ density $\mathrm{kg} \mathrm{m}^{-3}$

$\Phi \quad$ set of indices of system vertices

$\Omega \quad$ convex set of uncertain vertex systems

\section{Subscripts}

$0 \quad$ initial value

c cooling medium

$j \quad$ index of control input

$k \quad$ control step

max maximal value

min minimal value

$\mathrm{m}^{2}$ in input value

PO propylene oxide

PG propylene glycol

$\mathrm{r} \quad$ reaction mixture specific heat capacity $\quad \mathrm{kJ} \mathrm{kg}^{-1} \mathrm{~K}^{-1}$

\section{Superscripts}

NSOA nominal system optimization approach s steady state

WCSOA worst-case system optimization approach

$\mathrm{T}$ transposition of the matrix

(v) index of uncertain system vertex

(w) index of uncertain system vertex

\section{References}

Ball R, Gray BF (2013). Thermal instability and runaway criteria: The dangers of disregarding dynamics. Process Safety and Environmental Protection, 91: 221-226, DOI:1016/j.psep.2012.05.008.

Bakošová M, Mészáros A, Klemeš J, Oravec J (2012). Robust and Optimal Control Approach for Exothermic Reactor Stabilization. Theoretical Foundations of 
Chemical Engineering, 46: 740-746, DOI:10.1134/ S0040579512060036.

Bakošová, M, Oravec, J (2013). Robust MPC of a Chemical Reactor Using the Nominal System Optimization. In Proceedings of the 40th International Conference of Slovak Society of Chemical Engineering: 1070-1078. Tatranské Matliare, Slovakia.

Bakošová M, Oravec J, Matejíčková K. (2013). Model Predictive Control-Based Robust Stabilization of a Chemical reactor. Chemical Papers, 67: 1146-1156, DOI:10.2478/s11696-012-0296-2.

Cuzzola FA, Geromel JC, Morari, M. (2002). An improved approach for constrained robust model predictive control. Automatica: 38, 1183-1189.

Ding B. (2010). Constrained robust model predictive control via parameter-dependent dynamic output feedback. Automatica, 46: 1517-1523, DOI:10.1016/j. automatica.2010.06.14

Favache A, Dochain D (2010). Power-shaping control of reaction systems: The CSTR case. Automatica, 46: 1877-1883, DOI:10.1016/j.automatica.2010.07.011.

Ghaffari V, Naghavi, SV, Safavi AA (2013). Robust model predictive control of a class of uncertain nonlinear systems with application to typical CSTR problems. Journal of Process Control, 23: 493-499, DOI:10.1016/j.procont.2013.01.009.

Graichen K, Hagenmeyer V, Zeitz M (2009). Design of adaptive feedforward control under input constraints for a benchmark CSTR based on a BVP solver. Computers and Chemical Engineering, 33: 473-483, DOI:10.1016/j.compchemeng.2008.11.002.

Kothare MV, Balakrishnan V, Morari M (1996). Robust constrained model predictive control using linear matrix inequalities. Automatica, 32: 1361-1379.

Kvasnica M, Herceg M, Čirka L, Fikar M (2010). Model predictive control of a CSTR: A hybrid modeling approach. Chemical papers, 64: 301-309, DOI:10.2478/s11696-010-0008-8
Laššák P, Labovský J, Jelemenský L' (2010). Influence of parameter uncertainty on modeling of industrial ammonia reactor for safety and operability analysis. Journal of Loss Prevention in the Process Industries, 23: 280-288, DOI:1016/j.jlp.2009.10.001.

Löfberg J (2004). Yalmip: A toolbox for modelling and optimization in Matlab. Proc. of the CACSD Conference, Taipei, Taiwan, from http://control. ee.ethz.ch/joloef/yalmip.php: 284-289.

Molnár A, Markoš J, Jelemenský L', (2002). Accuracy of mathematical model with regard to safety analysis of chemical reactors. Chemical Papers, 56: 357-361.

Oravec J, Bakošová M (2012). Robust Constrained MPC Stabilization of a CSTR. Acta Chimica Slovaca: 153-158, DOI:10.2478/v10188-012-0023-1.

Qin SJ, Badgwell TA (2003). A survey of industrial model predictive control technology. Control Engineering Practice, 11: 733-764.

Sturm J (1999). Using SeDuMi 1.02, a MATLAB toolbox for optimization over symmetric cones. Optimization Methods and Software: 11-12, 625-653.

Švandová Z, Markoš J, Jelemenský L' (2005). HAZOP Analysis of CSTR with the Use of Mathematical Modelling. Chemical Papers, 59: 464-468.

Vásquez-Bahena J, Montes-Horcasutas MC, OrtegalLópez J, Magaña Plaza LBF (2004). Multiple steady-states in a continuous stirred tank reactor: an experimental case study for hydrolysis of sucrose by invertase. Process Biochemistry, 39: 2179-2182.

Wu F. (2001). LMI-based robust model predictive control and its application to an industrial CSTR problem. Journal of Process Control: 11, 649-659. 